\title{
Computationally Efficient Symbol-Level Precoding Communications Demonstrator
}

\author{
J.C. Merlano-Duncan, Jevgenij Krivochiza, Stefano Andrenacci, Symeon Chatzinotas, Björn Ottersten \\ SnT - securityandtrust.lu, University of Luxembourg, Luxembourg \\ Email: \{juan.duncan, jevgenij.krivochiza, stefano.andrenacci, symeon.chatzinotas, bjorn.ottersten\}@uni.lu
}

\begin{abstract}
We present a precoded multi-user communication test-bed to demonstrate forward link interference mitigation techniques in a multi-beam satellite system scenario which will enable a full frequency reuse scheme. The developed test-bed provides an end-to-end precoding demonstration, which includes a transmitter, a multi-beam satellite channel emulator and user receivers. Each of these parts can be reconfigured accordingly to the desired test scenario. Precoded communications allow full frequency reuse in multiple-input multiple-output (MIMO) channel environments, where several coordinated antennas simultaneously transmit to a number of independent receivers. The developed real-time transmission test-bed assist in demonstrating, designing and benchmarking of the new Symbol-Level Precoding (SLP) techniques, where the data information is used, along with the channel state information, in order to exploit the multi-user interference and transform it into useful power at the receiver side. The demonstrated SLP techniques are designed in order to be computationally efficient, and can be generalized to others multi-channel interference scenarios.
\end{abstract}

\section{INTRODUCTION}

Multi-User MIMO (MU-MIMO) beamforming, also know as Precoding, has been studied in previous years as a way to reduce the co-channel interference in the forward links in the wireless communications (Wi-Fi, LTE) and multi-beam satellite systems [1]-[4]. Wide broadband services through high throughput satellite (HTS) systems motivated the research of advanced signal processing techniques for interference mitigation to provide a better spectrum utilization with a reasonable complexity. MU-MIMO communication techniques are also promissory in other multi-channel interference scenarios such as in the case of Asymmetric digital subscriber line [5] (ADSL) and Powerline communications [6]. The approach to this challenge is the use of the aforementioned precoding techniques, which allow aggressive reuse of the frequency spectrum by exploiting the spatial multiplexing. The conventional channel-based Precoding techniques use the knowledge of the Channel State Information (CSI) in order to generate the transmitted precoded signals. The most common channelbased strategies are the channel inversion, also known as Zero Forcing (ZF), and the Minimal Means Square Error (MMSE) method [7].

978-1-5386-3531-5/17/\$31.00 (c) 2017 IEEE
More advanced approaches also use the knowledge of the intended transmitted symbols, in addition to the CSI, in order to obtain an optimal set of precoded symbols per each transmission time slot. The challenge behind hardware implementations is to design realistically feasible precoder algorithm in terms of computational complexity, while facing real impairments over the communication link. Despite that numerous theoretical publications on symbol-level precoding techniques have appeared recently [8]-[10], practical implementation was out of their scope. Last year, the SIGCOM (Signal Processing) Research group from the University of Luxembourg has started to build a Satellite Precoding Hardware demonstrator for full frequency reuse named SERENADE [11]. The demonstrator includes symbol-level precoding (SLP) techniques which optimize the beamforming vectors per every transmitted set of symbols. This leads to improving Signal-to-overall-Noise ratio, service availability and energy efficiency. In this paper, we demonstrate the feasibility of SLP techniques in a practical hardware implementation. Towards this objective, we describe the practical implementation aspects of the hardware demonstrator, which consist of: a MIMO transmitter, which implements the SLP, a MIMO satellite channel emulator, and a set of receivers with CSI estimation capabilities. We integrate precoding techniques in the transmitter and benchmark performance in close-to-reality conditions to demonstrate proof-of-concept technique for full frequency reuse for HTS and further improve performance of the system. We developed practical optimization of the precoded transmission algorithms and derived the optimal solution for the design of the SLP precoder. The rest of this paper is organized as follows. In Section II, we state the system model, describe the precoding algorithms and analyze the computational efficiency. In Section III, we present experimental validation and analyze the experimental results of the precoding algorithm. Finally, we draw conclusions in Section IV. Notation: Upper-case and lower-case bold-faced letters are used to denote matrices and column vectors. The superscripts $(\cdot)^{H}$ and $(\cdot)^{-1}$ represents Hermitian matrix and inverse operations. $\|\cdot\|_{2}$ is the Euclidean norm, $|\cdot|$ is the absolute value of a complex, $\mathbf{0}$ is the all zero vector, and trace is 
the sum of all diagonal elements of a matrix.

\section{Symbol-Level Precoding Design}

\section{A. System Model}

The general system model focuses on the forward link of a multi-beam satellite system, which aims at reusing the total available bandwidth among all beams of the coverage (the so-called full frequency reuse). We define as $N_{t}$ the number of transmitting antenna elements and $N_{u}$ the total number of users in the coverage area. In the specified MIMO channel model, the received signal at the $\mathrm{i}$-th user is $y_{i}=\mathbf{h}_{i}^{\dagger} \mathbf{x}+n_{i}$, where $\mathbf{h}_{i}^{\dagger}$ is a $1 \times N_{t}$ vector representing the complex channel coefficients between the $i$-th user and the $N_{t}$ antennas of the transmitter, $\mathbf{x}$ is defined as the $N_{t} \times 1$ vector of the transmitted symbols at a certain symbol period and $n_{i}$ is the independent complex circular symmetric (c.c.s.) independent identically distributed (i.i.d) zero mean Additive White Gaussian Noise (AWGN) measured at the $i$-th user's receive antenna.

Assuming a system having $N_{t}=N_{u}=N$, which is the present case, looking at the general formulation of the received signal, which include the whole set of users, the linear signal model is:

$$
\mathbf{y}=\mathbf{H x}+\mathbf{n}=\mathbf{H W} \mathbf{s}+\mathbf{n}
$$

where $\mathbf{y}$ and $\mathbf{n} \in \mathbb{C}^{N}, \mathbf{x} \in \mathbb{C}^{N}$ and $\mathbf{H} \in \mathbb{C}^{N \times N}$. In this scenario, we define the linear precoding matrix $\mathbf{W} \in$ $\mathbb{C}^{N \times N}$ which maps information symbols $\mathbf{s}$ into precoded symbols $\mathrm{x}$.

\section{B. Precoding technique}

We implemented low-complexity symbol-level precoding technique [12] into the demonstrator. The algorithm aims to minimize total power of precoded symbols while sustaining a minimal SNR requirement of a received signal. The method optimally preserves constructive interference components to decrease the total consumed power at the transmitter side. The essential difference from a linear precoding method is the optimization vector $\left(\mathbf{u} \in \mathbb{C}^{N}\right)$, which is recalculated per every symbol set (s) to construct optimal precoded signal

$$
\mathbf{x}=\mathbf{W}_{\mathrm{ZF}}(\mathbf{s}+\mathbf{u}),
$$

where $\mathbf{W}_{\mathrm{ZF}}=\hat{\mathbf{H}}^{H} \cdot\left(\hat{\mathbf{H}} \cdot \hat{\mathbf{H}}^{H}\right)^{-1}$ is Zero-Forcing precoding matrix, $\hat{\mathbf{H}}$ - a channel matrix estimated from CSI. The precoding technique maintains the minimal SNR of the received symbols as

$$
\begin{aligned}
& \min _{\mathbf{u}}\|\mathbf{x}\|_{2} \\
& \text { s. t. }|\mathbf{y}| \geq|\mathbf{s}|,
\end{aligned}
$$

for $\mathbf{H W}_{\mathrm{ZF}}=\mathbf{I}$ and $\mathbf{n}=\mathbf{0}$. It was shown in [12], that the problem (3) can be transformed into a non-negative least squares (NNLS) problem and solved for the vector $\mathbf{u}$. If a solution for a particular channel matrix can not be found, then $\mathbf{u}=\mathbf{0}$ and (2) turns into conventional Zero-Forcing precoding [7]. Therefore, the minimal performance of the proposed precoding technique is expected at a level of Zero-Forcing using statistically averaged CSI data. We will refer to the proposed precoding technique as NNLSSLP further in the paper.

\section{Non-Negative Least Squares algorithm}

The low-complexity SLP design, described in the previous section, brings hardware and software implementation towards an unified solution. The key to this is an efficient algorithm to solve the NNLS optimization problem. It can be implemented using a Software Defined Radio and FPGA platforms with a reasonable level of complexity. We use a standard fast nonnegativity-constrained least squares algorithm (FNNLS), presented in [13]. We implemented the algorithm in LabVIEW Communication code to provide the best computational performance. The most time consuming operation of such algorithm is solving of unconstrained linear least squares sub-problems via $\mathrm{QR}$ decomposition. The asymptotic complexity of the QR decomposition of a square matrix $\left(\mathbb{R}^{n \times n}\right)$ is $O\left(n^{3}\right)$. However, there are more efficient methods that can reduce considerably this level of complexity up to $O\left(n^{2}\right)$ [14].

\section{Impact of proposed SLP on constellation}

Figure 1 shows how one symbol of the QPSK constellation can have an amplitude excursion in the horizontal or the vertical axis with the proposed NNLS-SLP algorithm. Here we obtain a theoretical BER expression

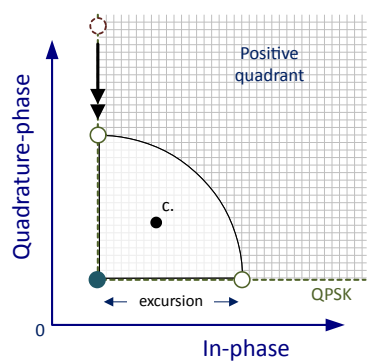

Fig. 1. Symbol excursion in NNLS-SLP. The symbol excursion can be in the vertical or horizontal axis

assuming that the receiver recovers perfectly the phase of the reference symbols. This phase recovery may be approached in a realistic scenario by means of repetitive pilot symbols (precoded or not) which are not modified. In addition, an accurate synchronization can be maintained if modified symbols have in average the same phase of the mapping symbols. For the particular case of a symbol of a QPSK modulation, with an excursion ratio $\epsilon$ of the mapping symbol, which can be in the inphase or quadrature axes, the BER is $p_{\text {es }}=0.5(\mathcal{Q}(\sqrt{\gamma})+$ $\mathcal{Q}(\sqrt{\gamma}(1+\epsilon)))$ where $\mathcal{Q}(\cdot)$ is the standard Gaussian complementary cumulative distribution function, and $\gamma$ 
is the SNR, where we assume that the received signal is affected by an additive zero-mean circulary-symetric complex Gaussian noise. The ensemble BER is computed to be $0.75 \mathcal{Q}(\sqrt{\gamma})+0.25 \mathcal{Q}(\sqrt{\gamma}(1+\epsilon))$, under the assumption that all symbols have the same probability and half of the symbols have the same amplitude excursion in one dimension and the other half do not have any excursions.

\section{EXPERIMENTAL VALIDATION}

In order to demonstrate the feasibility of the proposed SLP technique we build a $2 \times 2$ demonstrator with a transmitter, a channel emulator and two receivers using SDR platforms. Figure 2 shows a simplified block diagram of the Precoding demonstrator. The transmitter performs the

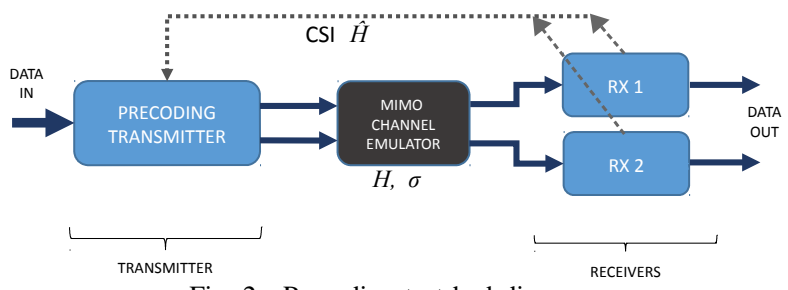

Fig. 2. Precoding test bed diagram.

precoding operation using the CSI obtained by means of a dedicated return channel. The transmitted signals are sent to the MIMO channel emulator, which apply the channel matrix $\mathbf{H}$ and inject to the signals an additive white Gaussian noise with a controlled power. These channel functionalities are implemented in a FPGA which is integrated to the SDR platform. The RF inputs and outputs of the channel emulator operate at different carrier frequencies. Using this configuration we decrease mutual coupling between the transmission and reception links through the RF part of the channel emulator and therefore the accuracy in setting of the desired channel matrix. Table I shows a summary of the parameters of the precoded transmitted signals.

TABLE I

EXPERIMENTAL PARAMETERS OF PRECODED TRANSMISSION IN 2X2 MIMO SYSTEM

\begin{tabular}{|l|c|}
\hline Parameter & Value \\
\hline Modulation type & QPSK \\
TX to Emulator c. frequency & $1210 \mathrm{MHz}$ \\
Emulator to RXs c. frequency & $960 \mathrm{MHz}$ \\
Sampling frequency & $1 \mathrm{MHz}$ \\
Over-sampling factor & 4 \\
Pulse shaping filter & SRRC with 0.2 roll-off \\
Pilot duration & 24 symbols \\
Data duration & 896 symbols \\
Pilot repetition period & 2048 symbols \\
\hline
\end{tabular}

Before precoding, each of the input bit streams are xor-scrambled with different gold sequences obtained from the combinations of the two maximum-length sequences with the characteristic polynomials $1+x^{3}+x^{20}$ and $1+x^{2}+x^{11}+x^{17}+x^{20}$. This scrambling is used in order to obtain a transmission in which all the symbols have the same probability of occurrence. The transmitted data is a set of two different video streams that are recovered at the receivers. Figure 3 shows a photography of the experimental set-up. We use National Instruments USRP-RIO NI-2944-R as SDR platform. Each of the SDR platforms is controlled by a dedicated PC host. The PCs are also used for data collection, processing and visualization. The channel emulator can generate $2 \times 2$

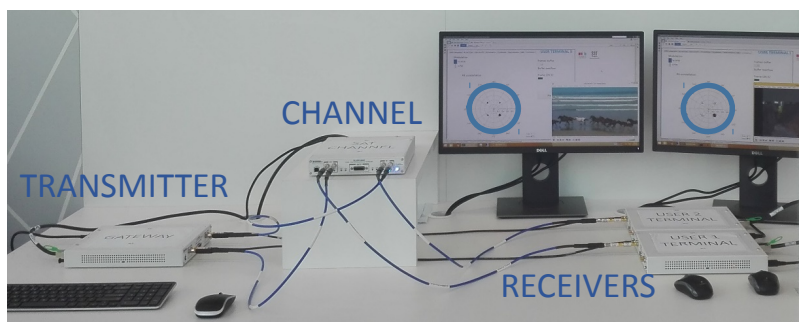

Fig. 3. Precoding $2 \times 2$ Experimental Settings. The SDR platform used for transmitter, channel emulator and receivers is the NI-2944-R.

complex channel matrix with a given condition number and set accurately the power of the AWGN. We as well use the channel emulator to measure actual transmission power on each port of the transmitter.

\section{A. Symbol-Level Optimized Precoding Evaluation}

We use aforementioned experimental environment to benchmark the optimized symbol-level precoding technique. We generate a set of random channel matrices $\mathbf{H}$ with unitary matrix F-norm, defined by $\|\mathbf{H}\|_{\mathbf{F}}=$ trace $\left(\mathbf{H}^{H} \mathbf{H}\right)$, and for different matrix conditioning numbers, defined by:

$$
\kappa_{2}(\mathbf{H})=\|\mathbf{H}\|_{2} \cdot\left\|\mathbf{H}^{-\mathbf{1}}\right\|_{2} .
$$

We apply the NNLS-SLP and compare the results to conventional channel-inversion ZF precoding. In both cases we normalize the precoding matrix to have an unitary 2-norm, so that we obtain a constant value for the expectancy of transmitted power per antenna. Under this constraints we measured the power in the two receivers and compare the results for different channel realizations for a set of channel matrix conditioning numbers between 2.5 and 4, as is shown in Figure 4. It is worth to note that in both cases ZF and NNLS-SLP we use the same channel inversion matrix. However, the difference for NNLS-SLP is the use of optimized symbols, which are limited to the unitary amplitude. From Figure 4 we can observe that the received power for ZF precoding is not a constant for a given conditioning number as should be expected from the theory. These variations come from the imperfections in the actual hardware implementation. Some of these imperfections are the limited accuracy in the CSI estimation, and its quantization error. Here, we can observe the gains in received power for the NNLSSLP. These gains become more frequent as the matrix 


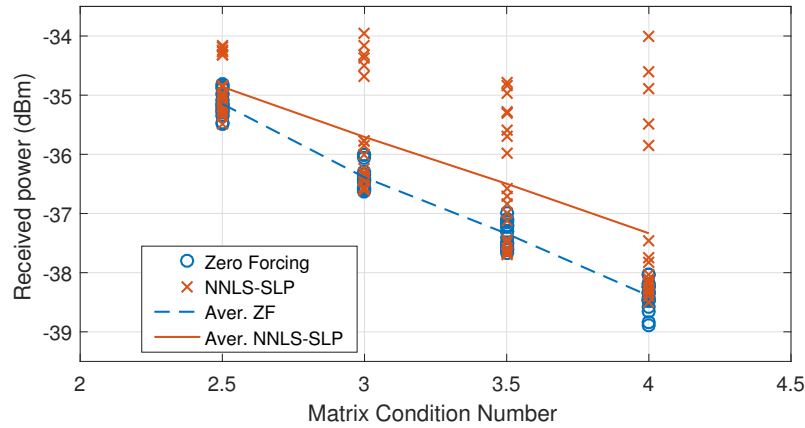

Fig. 4. Different realizations of detected power, in 2 receivers indistinctly, for conventional ZF and NNLS-SLP.

conditioning number is increased. There are particular channel realization in which the NNLS-SLP result is the same of ZF for both receivers, and others realization in which the optimized symbol is only produced for one of the receivers. Up to this point, we have observed the gains in received power for NNLS-SLP. In the following we will observe how this gain is translated to BER performance in the receiver.

\section{B. Bit Error Performance of NNLS-SLP}

Figure 5 shows an example of received modified constellation with the NNLS-SLP algorithm with some AWGN already applied. This constellation will be difficult to demodulate by a conventional QPSK demodulator as the one used (Labview Communications v.2.0), as the phase synchronization algorithm tracks for the mean square phase error, which is increased in the proposed constellation. However, the symbol excursion will help for the cases in which the phase is correctly recovered and also for very low SNR conditions in which the received signal is very affected by additive noise. For this reason we proceed to perform BER experiments for different SNR values. The SNR is set

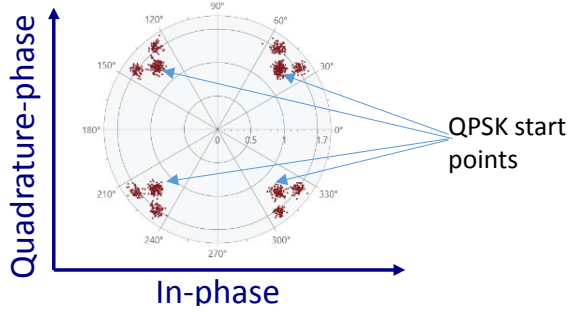

Fig. 5. An example of NNLS-SLP modified received constellation.

by means of the injection of artificial AWGN in the channel emulator. The noise power can be accurately controlled to adjust the desired SNR, knowing the exact value of the received signal power. First, we performed a single link BER measurement using an unmodified QPSK constellation. We use it as a reference to evaluate the effects of imperfect phase synchronization for low SNR values. Phase-locked loop of the demodulator is

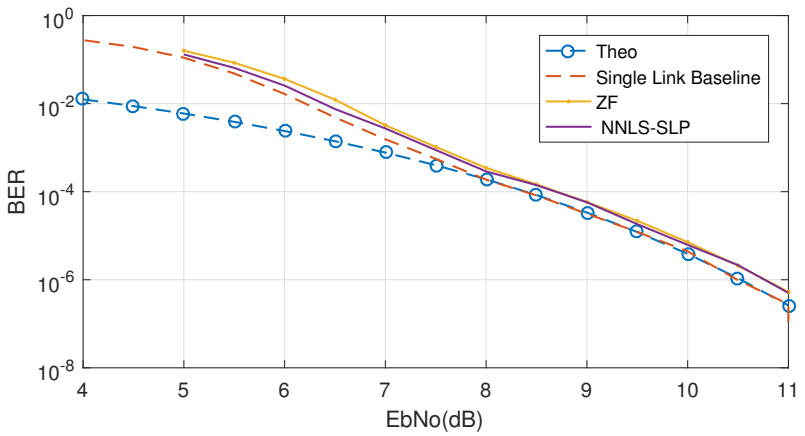

Fig. 6. Experimental BER plots for ZF and NNLS-SLP compared to a experimental baseline non-interference QPSK BER and to the theoretical BER curve. The matrix condition for the precoded channel is 2.5. The NNLS-SLP in this case provides an excursion of $4 \%$. Only a slight degradation is seen in the precoded system, which carries twice the data rate using the same frequency band.

reset for every frame. For the case of NNLS-SLP the precoded pilot symbols are not modified from the QPSK original mapping points. We performed measurements of BER for ZF and NNLS-SLP for different channel matrices, where the SNR was estimated solely using ZF precoding. This is a fair comparison, since, despite the average received power can increase while using NNLSSLP, minimal received power can still match the one gained with ZF precoding. Figure 6 shows the theoretical ideal QPSK BER values, the BER for a single noninterference link, and the BER for ZF and NNLS-SLP for a particular matrix with conditioning number 2.5 which gives and excursion (in horizontal and vertical axis) of $4 \%$. Here we see a degradation of the BER plots which use precoding compared to the single link BER curve. This is attributed to the inaccuracies in the CSI estimation, which produces residual interference that affects the BER performance, however, we should remark that for the case of precoded signals we obtain twice spectral efficiency, since the system provide two separate streams using the same frequency band. In the comparison between the ZF and NNLS-SLP we shown that the NNLS-SLP perform better for low SNR values, and that the ZF perform better at some points of higher SNR values using conventional receiver. The experiment is repeated with some channel matrices with higher proposed excursion values which in some cases gives a degradation in BER performance for high SNR values. Most of these errors are attributed to lack of phase synchronization and phase tracking. These effects can be observed as a rotated shaking in the constellation plots in the graphical user interface at the receiver. It is worth to clarify that, at the transmitter QPSK modulator maps the transmitted symbols in correspondence to optimization excursion. However at the receiver QPSK demodulator normalizes the received symbols in correspondence to conventional QPSK symbol map only as receiver has no 


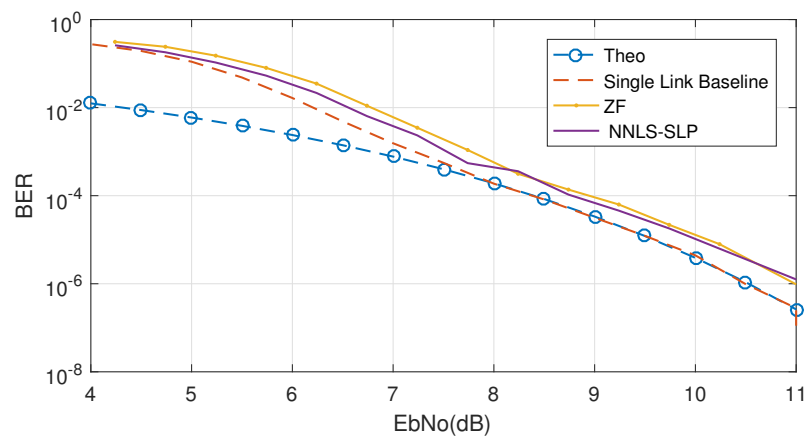

Fig. 7. Experimental BER plots for ZF and NNLS-SLP compared to a experimental baseline QPSK BER and to the theoretical BER curve. The matrix condition number for the precoded channel is 3 The NNLS-SLP in this case provides an excursion of $20 \%$.

knowledge about optimized mapping. However, at the receiver the QPSK start points are recovered in their original amplitude position, which is the same amplitude obtained when the ZF precoding is applied. Figure 7 shows the BER curves for for ZF and NNLS-SLP for a particular matrix with conditioning number of 3 which gives and excursion (in horizontal and vertical axis) of $20 \%$. Here we can observe how the NNLS-SLP performs better than the conventional $\mathrm{ZF}$ for $\mathrm{Eb} / \mathrm{No}$ values lower than $8 \mathrm{~dB}$.

\section{CONCLUSIONS}

In this paper, we presented the results of the hardware demonstration of precoded satellite communications based on state-of-art SLP technique. The SLP beamforming benchmarked similar BER results to $\mathrm{ZF}$ beamforming in the case scenario of high SNR. However, it is shown that SLP technique takes advantages of unbalanced channel matrices with strong interference components to increase received signal SNR.Therefore, we evidently shown that SLP technique can provide better SNR performance than conventional ZF technique in real-time transmissions for low SNR scenarios. A decrease in the BER performance for high SNR scenarios was observed. This performance decrease, which is generated by an imperfection in the carrier synchronization and tracking can be avoid if global synchronization is achieved. Some of examples of this are the multi-carrier or OFDM systems in which many carriers are jointly synchronized and aided by additional synchronization carrier pilots. Another example in which this method can be applied is in the low SNR regimes of single carrier communications which maintain phase coherency along time, such as the satellite communication standards as DVB-S2X, where the receiver synchronization is improved over longer averaging periods. In addition, the improvements for the evaluated method will increase with higher order MIMO systems, where the gains obtained from the symbol-level optimization can increase. In this paper we also show, to the best author knowledge, the first symbol-level optimized precoded communication system, in a real hardware implementation, where we show the feasibility in terms of complexity of the proposed SLP optimized method.

\section{ACKNOWLEDGEMENTS}

This research was supported by Luxembourg National Research Fund grant for "SERENADE" project and AFR-PPP "End-to-end Signal Processing Algorithms for Precoded Satellite Communications" project.

\section{REFERENCES}

[1] W. Zeng, C. Xiao, M. Wang, and J. Lu, "Linear precoding for finite-alphabet inputs over mimo fading channels with statistical csi," IEEE Transactions on Signal Processing, vol. 60, no. 6, pp. 3134-3148, June 2012.

[2] N. Song, T. Yang, and M. Haardt, "Efficient hybrid space-ground precoding techniques for multi-beam satellite systems," in 2017 IEEE International Conference on Acoustics, Speech and Signal Processing (ICASSP), March 2017, pp. 6284-6288.

[3] Q. H. Spencer, A. L. Swindlehurst, and M. Haardt, "Zero-forcing methods for downlink spatial multiplexing in multiuser mimo channels," IEEE Transactions on Signal Processing, vol. 52, no. 2, pp. 461-471, Feb 2004.

[4] X. Artiga, M. A. Vazquez, A. Perez-Neira, C. Tsinos, E. Lagunas, S. Chatzinotas, V. Ramireddy, C. Steinmetz, R. Zetik, K. Ntougias, D. Ntaikos, and C. B. Papadias, "Spectrum sharing in hybrid terrestrial-satellite backhaul networks in the ka band," in 2017 European Conference on Networks and Communications (EuCNC), June 2017, pp. 1-5.

[5] R. B. Moraes, P. Tsiaflakis, J. Maes, and M. Moonen, "General framework and algorithm for data rate maximization in dsl networks," IEEE Transactions on Communications, vol. 62, no. 5, pp. 1691-1703, May 2014.

[6] S. Neshvad, S. Chatzinotas, and J. Sachau, "Wideband identification of power network parameters using pseudo-random binary sequences on power inverters," IEEE Transactions on Smart Grid, vol. 6, no. 5, pp. 2293-2301, Sept 2015.

[7] C. B. Peel, B. M. Hochwald, and A. L. Swindlehurst, "A vectorperturbation technique for near-capacity multiantenna multiuser communication-part i: channel inversion and regularization," IEEE Transactions on Communications, vol. 53, no. 1, pp. 195202, Jan 2005.

[8] Ashkan Kalantari, Christos G. Tsinos, Mojtaba Soltanalian, Symeon Chatzinotas, Wing-Kin Ma, and Björn E. Ottersten, "Energy-efficient M-QAM precoder design with spatial peak power minimization for MIMO directional modulation transceivers," CoRR, vol. abs/1702.06878, 2017.

[9] Y. C. B. Silva and A. Klein, "Linear Transmit Beamforming Techniques for the Multigroup Multicast Scenario," IEEE Transactions on Vehicular Technology, vol. 58, no. 8, pp. 4353-4367, Oct 2009.

[10] C. Masouros and E. Alsusa, "Dynamic linear precoding for the exploitation of known interference in MIMO broadcast systems," IEEE Transactions on Wireless Communications, vol. 8, no. 3, pp. 1396-1404, March 2009.

[11] Prof. Bjorn Ottersten, Dr. Symeon Chatzinotas, Dr. Stefano Andrenacci, Dr. Juan Merlano Duncan, and Dr. Maha Alodeh, "Serenade: Satellite precoding hardware demonstrator," 2017.

[12] J. Krivochiza, A. Kalantari, S. Chatzinotas, and B. Ottersten, "Low complexity symbol-level design for linear precoding systems," in 2017 Symposium on Information Theory and Signal Processing in the Benelux. Delft University of Technology, 2017, p. 117.

[13] Rasmus Bro and Sijmen De Jong, "A Fast Non-negativityconstrained Least Squares Algorithm," Journal of Chemometrics, vol. 11, no. 5, pp. 393-401, 1997.

[14] Yuancheng Luo and Ramani Duraiswami, "Efficient parallel nonnegative least squares on multicore architectures," SIAM Journal on Scientific Computing, vol. 33, no. 5, pp. 2848-2863, 2011. 